\title{
Alterations in the immunoreactivity of laminin, type IV collagen and $\alpha 3 \beta 1$ integrin in diabetic rat ovarian follicles
}

\author{
Yildirim AB ${ }^{1}$, Karabulut D ${ }^{2}$, Ozturk E ${ }^{2}$, Kaymak E ${ }^{3}$, Yalcin $B^{2}$, Kuloglu N ${ }^{4}$, Akkus E \\ Gaziantep Islam, Science and Tecnology University, Department of Histology and Embryology, \\ Gaziantep, Turkey. aysegulburcin@gmail.com
}

\begin{abstract}
AIM: In order to determine the possible effects of diabetes, we aimed to investigate the expression of extracellular matrix proteins in the theca and granulosa layers in different follicular stages.

METHODS: Thirty-two adult Wistar albino male rats were divided into 4 groups as control and sampled groups. Four, eight and twelve weeks after inducing diabetes with an intraperitoneal injection of streptozotocin (40 mg/kg), the expressions of laminin, type IV collagen and $\alpha 3 \beta 1$ integrin in ovarian tissues were evaluated by immunohistochemical method.

RESULTS: In our study, in the first month of diabetes, a significant increase was observed in laminin, type IV collagen and $\alpha 3 \beta 1$ integrin expressions in all follicle types compared to the control group in both the theca and granulosa layers. Laminin and type IV collagen immunoreactivity tended to increase in D2 and D3 groups also. Integrin expression did not change in the newly formed follicles in the D2 and D3 groups, however, it tended to change and increase in the developing follicles.

CONCLUSIONS: The changes in the expression of laminin, type IV collagen and $\alpha 3 \beta 1$ integrin, which are the extracellular matrix proteins in the follicle, along with diabetes, show that diabetes plays a role in the regulation of follicular development (Tab. 4, Fig. 36, Ref. 29). Text in PDF www.elis.sk.

KEY WORDS: $\alpha 3 \beta 1$ integrin, laminin, type IV collagen, diabetes, IHC, ovarian follicles.
\end{abstract}

\section{Introduction}

Diabetes mellitus is considered to be a metabolic disorder which is characterized by insufficient secretion or endogenous insulin action and hyperglycemia (1).

Diabetes is related with an increased risk of diseases such as cardiovascular disorders and neuropathy. At the same time however, it is associated with reproductive problems such as poor embryo development, congenital malformation, impaired folliculogenesis, spontaneous abortions, and anovulation in women $(2,3)$. Folliculogenesis is an extremely regulated process where various autocrine, endocrine and paracrine factors act spatially and temporally in order to coordinate and regulate the development and growth of the oocyte as well as the surrounding theca and granulosa cell layers (4). The extracellular matrix has different roles in the cellular development in the ovary and in many

${ }^{1}$ Gaziantep Islam, Science and Tecnology University, Department of Histology and Embryology, Gaziantep, Turkey, ${ }^{2}$ Erciyes University, Department of Histology and Embryology, Kayseri, Turkey, ${ }^{3}$ Bozok University, Department of Histology and Embryology, Yozgat, Turkey, ${ }^{4}$ Nigde Ömer Halisdemir University, Department of Health Care Services, Niğde, Turkey, and ${ }^{5}$ Nizip State Hospital, Gaziantep, Turkey

Address for correspondence: A.B. Yildirim, Ass. Prof. Dr., Gaziantep Islam, Science and Tecnology University, Department of Histology and Embryology, Gaziantep, Turkey.

Acknowledgement: This study was supported by Erciyes University Scientific Research Projects. systems. The extracellular matrix (ECM) provides the structural support to the follicle, provides biochemical signals which promote maturation and follicular development, as well as provides connectivity and cellular organization $(5,6)$. As the ovary is reshaped by continuous follicle growth, development and atresia, it is important to detect the cells in which ECM components are expressed at different stages of rat ovarian follicle. In addition, immunohistochemical studies conducted on the effect of diabetes on type IV collagen, laminin and $\alpha 3 \beta 1$ integrin expressions in these stages are very limited.

Laminin was localized to theca cell compartment primarily, with a characterized ring outside the follicular granulosa cells that mark the basement membrane. Lower levels of laminin were likewise clear in the granulosa, and stroma cell compartment. Collagen IV was found to be abundant in theca cell compartment, with a low-level expression in the granulosa and stroma cells. The distribution of collagen was consistent during the follicular development (4). Integrins are considered to be the major receptors which mediate adhesion to ECM (7). They are trans-membrane heterodimeric glycoproteins that are composed of subunits $\alpha$ and $\beta ; 16$ different $\alpha$ and $8 \beta$ subunits have been determined (8), and according to $\beta$ subunits, they have been divided into subfamilies. The $\beta 1$ superfamily contains specific receptors for laminin. Some $\beta 1$ integrins may bind collagens or laminin. However, the most versatile receptor is the $\alpha 3 \beta 1$ integrin, which binds collagen molecules (9) to structures such as laminin (10) and fibronectin $(8,11,12)$. 
The follicular microenvironment varies significantly throughout folliculogenesis because of the follicle movement along the ovary, changing extracellular milieu and development of the follicle. The folliculogenesis may be regulated by ECM through the composition which changes with both cellular compartment and follicle stage (6). ECM components that are present in follicular fluid, follicular basement membrane and around the follicular cells of the ovary, take part in the follicular development regulation, and the role of integrins is highly suggested in this process (7). The determining of ECM protein distributions and understanding the changes of ECM with diabetes in different ovarian follicle stages in rats will contribute to the understanding of the possible effects of diabetes on follicles.

In this study, streptozotocin (STZ) was administered to 12-week-old female mice to establish the type 1 diabetes model. The aim of this study was to investigate the laminin, type IV collagen and $\alpha 3 \beta 1$ integrin expressions in theca and granulosa layers in different follicular stages using immunohistochemical methods in order to reveal the possible effects of diabetes on rat follicular development stages.

\section{Material and methods}

\section{Animals}

This study was conducted in the Erciyes University, Hakan Çetinsaya Experimental and Clinic Research Center. Thirty-two adult twenty-week-old Wistar albino rats were used in this study. The rats were placed in a well-ventilated plastic cage rat house, kept on a 12-h light and dark cycle, while the feed and water were provided ad libitum. All animals were given good care according to the standard guidelines. Ethical approval from the Erciyes University, Animal Research Ethics Committee was obtained for the study (no: 12/105/2012). Ethical regulations were strictly followed according to the national and institutional guidelines. The rats were assigned randomly to four groups of eight rats per group. Group C (control) served as control; groups D1, D2 and D3 were composed of diabetic rats according to the duration of diabetes, namely one, two or three months, respectively.

\section{Diabetes induction and tissue sampling}

Diabetes induction was performed in 12-week-old female Wistar rats after an overnight fast $(\mathrm{n}=24)$ using STZ $(40 \mathrm{mg} / \mathrm{kg})$ intraperitoneal injection (Sc-200719, Santa Cruz Biotechnology, CA, USA), while physiological saline $(n=8)$ was injected in the control rats. Seventy-two hours after the injection of streptozotocin, hyperglycemia was confirmed by measurements of glucose levels in the blood, which was obtained from the tail vein. The measurements were done by using a glucometer. Animals whose mean plasma glucose levels were higher than $250 \mathrm{mg} / \mathrm{dL}$, were considered to be diabetic. After streptozotocin injection, glycemia was checked as well at sacrifice, i.e. at weeks 4,8 or 12 . The animals were decapitated under intraperitoneal xylazine $(10 \mathrm{mg} / \mathrm{kg})$ + ketamine $(75 \mathrm{mg} / \mathrm{kg})$ anesthesia. The ovarian tissues were removed quickly after decapitation, and they were fixed in a fixative of $4 \%$ formaldehyde for histological examination.

\section{Immunohistochemistry}

The Avidin-Biotin-Peroxidase Complex (ABC) method and a couple of immunohistochemical methods were used to determine laminin, type IV collagen and $\alpha 3 \beta 1$ integrin immunoreactivities in the ovarian tissue. Paraffin sections ( $5 \mu \mathrm{m}$ thick) were placed on poly-(L-lysine)-coated slides and stored overnight in an oven at $60{ }^{\circ} \mathrm{C}$. Then they were deparaffinized with xylene and rehydrated with a series of decreasing grades of alcohol (100\%, $96 \%, 80 \%, 70 \%$ ). After rehydration, the sections were washed in distilled water 3 times for 5 minutes. For antigen retrieval, the tissues were heated for 5 minutes in $5 \%$ citrate buffer in a microwave oven at $600 \mathrm{~W}$. The sections were washed with phosphate buffered saline (PBS) and treated with $3 \%$ hydrogen peroxide $\left(\mathrm{H}_{2} \mathrm{O}_{2}\right)$ for 12 minutes in order to block endogenous peroxidase. For the next stages, a Large Volume Detection System (Thermo Fisher Scientific, Waltham, MA, USA, Catalog no: TP-125-HL) immunochemistry staining kit was used. To prevent background staining, the sections were treated with ultra $V$ block for 5 minutes and then incubated overnight at $+4{ }^{\circ} \mathrm{C}$ in a humid environment with appropriate primary antibodies (Laminin (Anti- Laminin antibody (abcam), ab11575, 1/100 dilution), Type IV collagen (Anti-Collagen IV antibody (abcam), ab6586, 1/100 dilution) and $\alpha 3 \beta 1$ integrin (Integrin Alpha3, Beta1 antibody, ABIN737034, 1/50 dilution). Negative controls were treated with PBS in place of the primary antibodies. Processes in the other stages mentioned above were carried out in the same way. After primary antibody incubation, the sections were rinsed. Reverse staining was performed using appropriate biotinylated secondary antibodies, Streptavidin-HRP (Horse Radish Peroxidase), DAB (3,3'-diaminobenzidine) chromogen, and Gill's Hematoxylin. Finally, the sections were dehydrated in a series of increasing grades of alcohol $(70 \%, 80 \%$, $96 \%, 100 \%$ ) and cleared in xylene before being cover-slipped with Entellan ${ }^{\circledR}$ (Merck, Kenilworth, NJ, USA) (13). The slides were viewed and photographed using an Olympus BX51 microscope with a digital camera (DP71).

\section{Evaluation of immunohistochemical staining}

Based on images that were taken from the slides, laminin, $\alpha 3 \beta 1$ integrin and type IV collagen immunoreactivity measurements were taken using Image-J program for immunoreactivity difference. Immunoreactivity differences were measured separately in granulosa and theca layers in each of the primary, secondary and tertiary follicles in all groups. Measurements were taken homogeneously from different follicles in each follicle type in the tissue. The data obtained were evaluated statistically.

Tab. 1. Blood glucose levels of control and streptozotocin (STZ)-induced diabetic rats.

\begin{tabular}{lc}
\hline Groups & Blood glucose $(\mathrm{mg} / \mathrm{dl})$ \\
\hline Group C & $118.87 \pm 2.18$ \\
Group D1 & $544.40 \pm 33.84^{*}$ \\
Group D2 & $517.25 \pm 42.30^{*}$ \\
Group D3 & $538.33 \pm 36.10^{*}$ \\
\hline * significantly differed from control, by $\mathrm{p}<0.05$ (according to ANOVA results)
\end{tabular}




\section{0-347}

\section{Follicle classification}

Follicles were classified in accordance with the commonly used terms for rats. Primary follicles contained one or fewer than two layers of the cuboidal granulosa cells. Secondary follicles (preantral follicles) had an oocyte and were categorized as twolayered or multi-layered secondary follicles according to the number of present granulosa cell layers, and had no visible antrum. In tertiary follicles (antral follicles), also, an oocyte, which might be surrounded by multiple layers of cuboidal granulosa cells and containing one or more cumuli oophori, antral spaces and theca layer, might have been evident $(6,14)$.

\section{Statistical analysis}

The obtained data were analyzed using SPSS 23.0. Firstly, to determine normality, the values of skewness and kurtosis were examined. ANOVA was used in order to determine statistical significance.
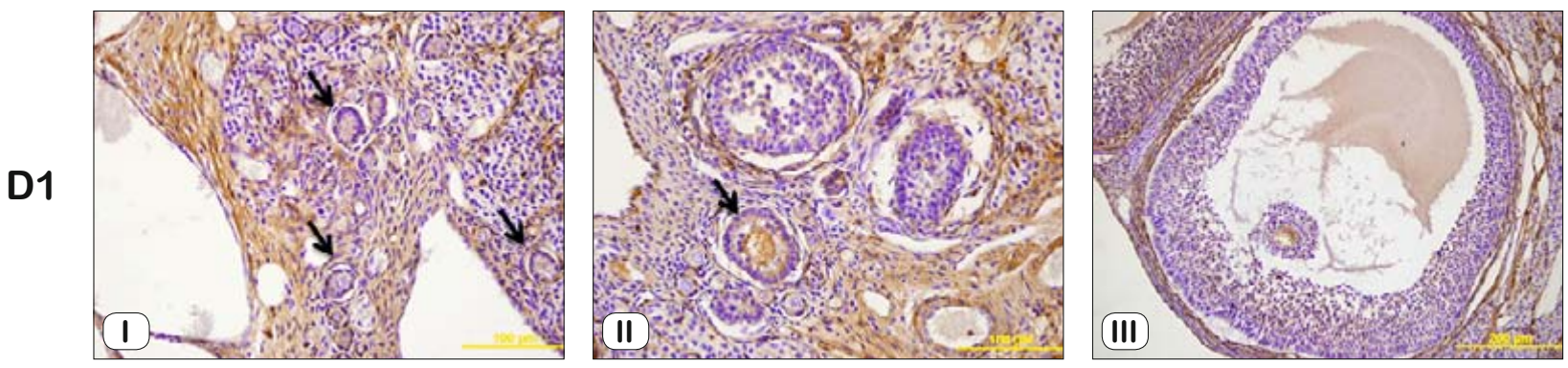

D2
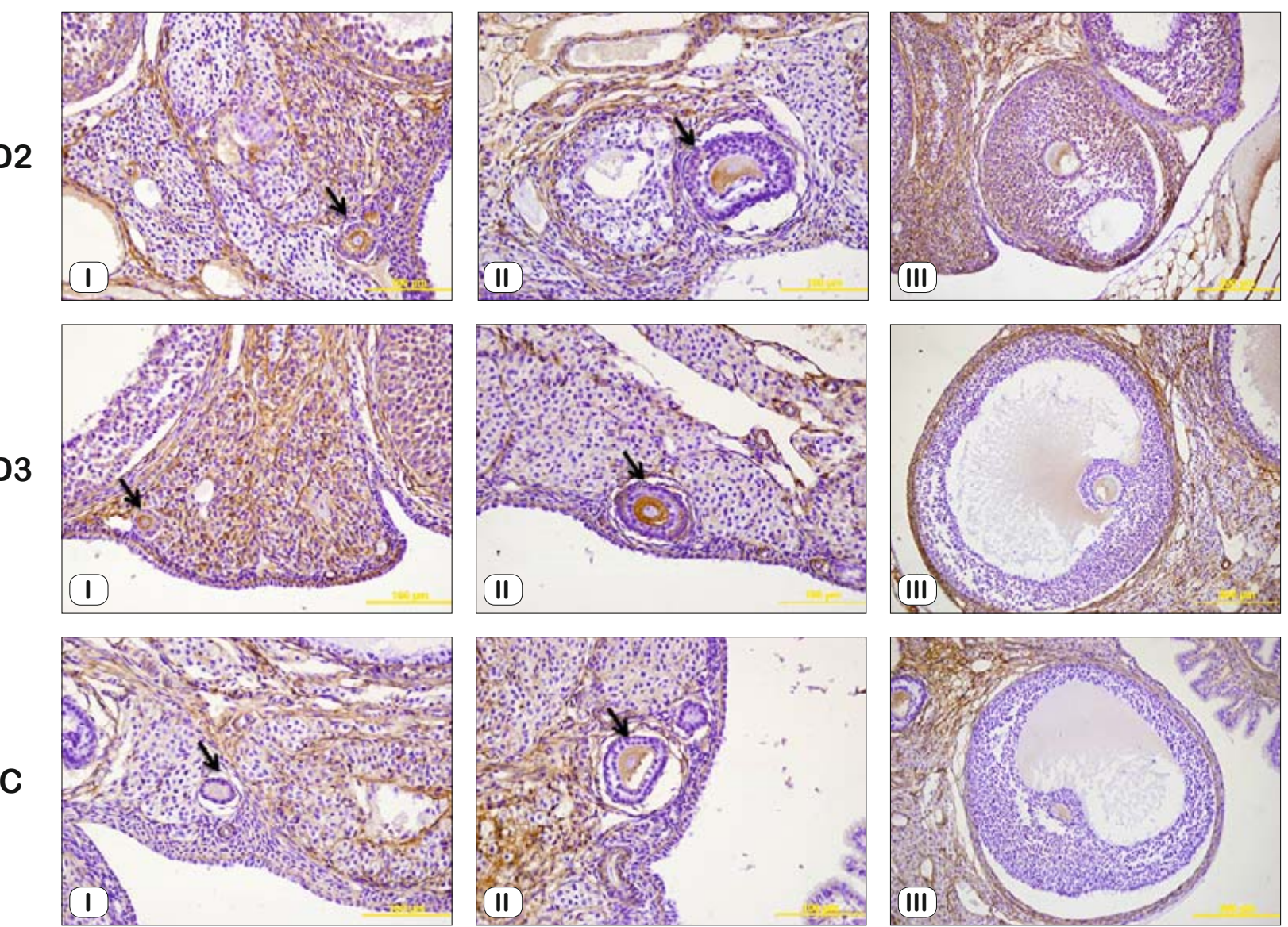

Fig. 1. Laminin immunostaining in experimental groups. Groups D1, D2, D3, and C: I (Primary Follicles), Laminin expression in the primary follicles (arrow), II (Secondary Follicles), Laminin expression in the secondary follicles (arrow), III (Tertiary Follicles). I, II X20, Bar 100 $\mu$ m; III X40, Bar $200 \mu \mathrm{m}$. 
Tab. 2. Immunoreactivity intensity values of Laminin in the follicles.

\begin{tabular}{llcccc}
\hline \multirow{2}{*}{ LAMININ } & & \multicolumn{3}{c}{$\overline{\mathrm{X}} \pm$ SD } \\
\cline { 3 - 6 } & & Control & D1 & D2 & D3 \\
\hline Primary fol. & Granulosa & $93.44 \pm 6.68$ & $103.58 \pm 10.08^{* *}$ & $102.22 \pm 5.22^{* *}$ & $100.81 \pm 8.43^{* *}$ \\
\hline \multirow{2}{*}{ Secondary fol. } & Granulosa & $94.80 \pm 4.73$ & $102.39 \pm 7.19^{* *}$ & $100.05 \pm 5.24^{* *}$ & $99.22 \pm 6.00^{* *}$ \\
& Theca & $103.16 \pm 5.29$ & $110.75 \pm 7.90^{* *}$ & $105.85 \pm 6.92$ & $104.57 \pm 7.83$ \\
\hline \multirow{2}{*}{ Tertiary fol. } & Granulosa & $90.82 \pm 4.23$ & $100.33 \pm 5.71^{* *}$ & $95.64 \pm 5.98^{* *}$ & $96.76 \pm 4.64^{* *}$ \\
& Theca & $106.51 \pm 5.54$ & $115.45 \pm 8.15^{* *}$ & $107.82 \pm 7.29$ & $110.28 \pm 9.02$ \\
\hline
\end{tabular}

* significantly differed from control, by $\mathrm{p}<0.05$ (according to ANOVA results)

** significantly differed from control, by $\mathrm{p}<0.01$ (according to ANOVA results)
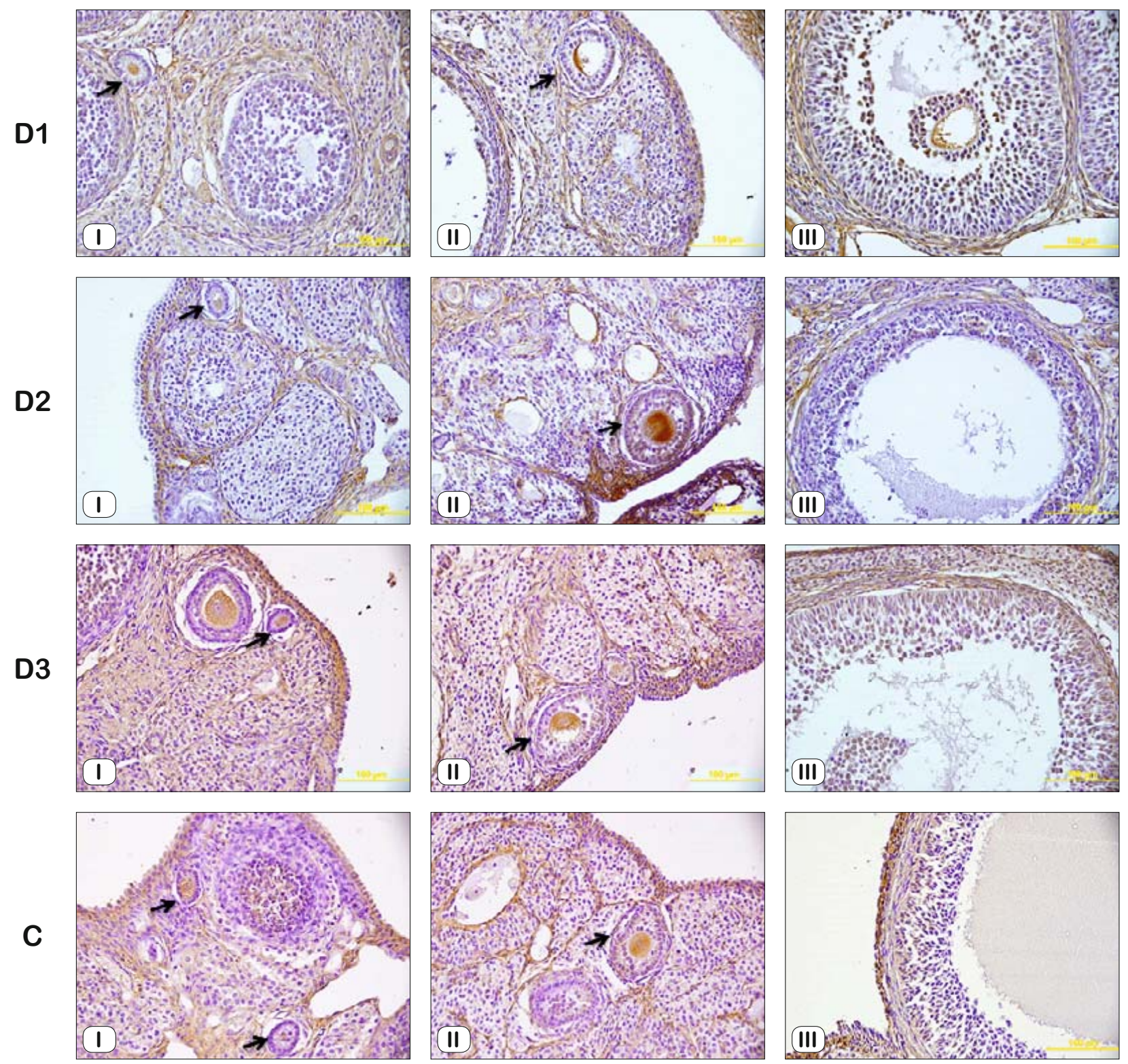

Fig. 2. Type IV collagen immunostaining in experimental groups. Groups D1, D2, D3, and C: I (Primary Follicles), Type IV collagen expression in the primary follicles (arrow), II (Secondary Follicles), Type IV collagen expression in the secondary follicles (arrow), III (Tertiary Follicles). I, II, III X40, Bar $100 \mu \mathrm{m}$. 
340-347

Tab. 3. Immunoreactivity intensity values of Type IV collagen in the follicles.

\begin{tabular}{|c|c|c|c|c|c|}
\hline \multirow{2}{*}{\multicolumn{2}{|c|}{ TYPE IV COLLAGEN }} & \multicolumn{4}{|c|}{$\overline{\mathrm{X}} \pm \mathrm{SD}$} \\
\hline & & Control & D1 & D2 & D3 \\
\hline Primary fol. & Granulosa & $90.93 \pm 7.28$ & $102.43 \pm 6.88 * *$ & $93.87 \pm 6.49$ & $96.54 \pm 5.79 * *$ \\
\hline \multirow{2}{*}{ Secondary fol. } & Granulosa & $92.14 \pm 7.11$ & $105.51 \pm 8.57 * *$ & $98.71 \pm 6.01 * *$ & $96.35 \pm 5.13^{*}$ \\
\hline & Theca & $93.29 \pm 5.68$ & $103.07 \pm 7.69 * *$ & $98.73 \pm 7.43 * *$ & $95.68 \pm 7.63$ \\
\hline \multirow{2}{*}{ Tertiary fol. } & Granulosa & $94.18 \pm 7.00$ & $108.23 \pm 7.37 * *$ & $104.05 \pm 6.63 * *$ & $103.33 \pm 7.78^{* *}$ \\
\hline & Theca & $100.07 \pm 8.76$ & $110.45 \pm 8.65^{* *}$ & $102.20 \pm 6.34$ & $104.32 \pm 10.19$ \\
\hline
\end{tabular}

* significantly differed from control, by $\mathrm{p}<0.05$ (according to ANOVA results)

** significantly differed from control, by $\mathrm{p}<0.01$ (according to ANOVA results)
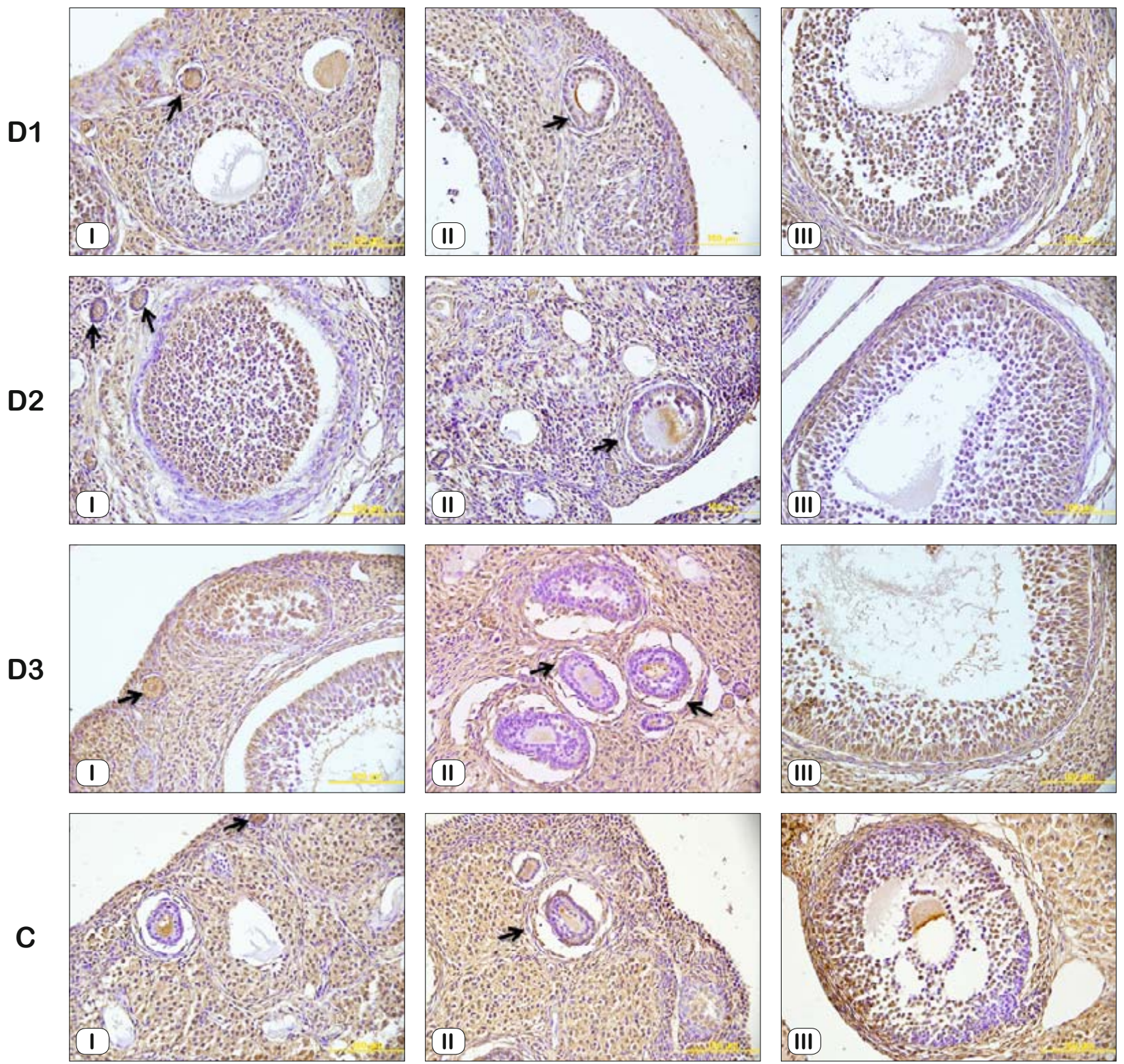

Fig. 3. Integrin $\alpha 3 \beta 1$ immunostaining in experimental groups. Groups D1, D2, D3, and C: I (Primary Follicles), $\alpha 3 \beta 1$ integrin expression in the primary follicles (arrow), II (Secondary Follicles), $\alpha 3 \beta 1$ integrin expression in the secondary follicles (arrow), III (Tertiary Follicles). I, II, III X40, Bar $100 \mu \mathrm{m}$. 
Tab. 4. Immunoreactivity intensity values of $\alpha 3 \beta 1$ integrin in the follicles.

\begin{tabular}{|c|c|c|c|c|c|}
\hline \multirow{2}{*}{$\alpha 3 \beta 1$ INTEGRIN } & & \multicolumn{4}{|c|}{$\overline{\mathrm{X}} \pm \mathrm{SD}$} \\
\hline & & Control & D1 & D2 & D3 \\
\hline Primary fol. & Granulosa & $96.01 \pm 9.67$ & $108.50 \pm 10.36^{* *}$ & $95.86 \pm 9.06$ & $97.81 \pm 13.09$ \\
\hline \multirow{2}{*}{ Secondary fol. } & Granulosa & $96.21 \pm 9.06$ & $103.66 \pm 7.31 * *$ & $93.87 \pm 9.90$ & $97.18 \pm 7.09$ \\
\hline & Theca & $92.45 \pm 5.71$ & $98.14 \pm 7.48 * *$ & $95.30 \pm 9.36$ & $94.11 \pm 9.48$ \\
\hline \multirow{2}{*}{ Tertiary fol. } & Granulosa & $96.55 \pm 5.43$ & $109.12 \pm 9.73 * *$ & $98.06 \pm 7.96$ & $103.47 \pm 7.77 * *$ \\
\hline & Theca & $94.21 \pm 4.69$ & $103.10 \pm 12.63 * *$ & $100.18 \pm 9.55^{*}$ & $100.61 \pm 10.24 * *$ \\
\hline
\end{tabular}

* significantly differed from control, by $\mathrm{p}<0.05$ (according to ANOVA results)

** significantly differed from control, by $\mathrm{p}<0.01$ (according to ANOVA results)

\section{Results}

\section{Induction of diabetes}

At the end of the experiment, blood glucose levels were measured. The increase in blood glucose levels were statistically significant in the diabetic groups as compared to the control group (Tab. 1).

\section{Immunohistochemistry}

Laminin immunoreactivity

Laminin was immunolocalized to granulosa cells, theca cell compartment and stroma for all the follicular stages (Fig. 1). According to the results of statistical analysis, laminin immunoreactivity in primary follicles containing granulosa cells showed a significant increase in D1, D2 and D3 groups as compared to the control group. There was a significant increase in granulosa layer of the secondary and tertiary follicles in D1, D2 and D3 groups compared to the control group. In the theca layer, a significant increase was observed in the D1 group, compared to the control group, whereas, there was no significant increase in the D2 and D3 groups compared to the control group. According to these data, as the diabetes progressed in rats, there was an increase in laminin immunoreactivity in granulosa cells in all follicular stages, whereas the immunoreactivity of the theca layer was observed to be increased in the initial stages of diabetes. In later stages however, it was observed to be similar to that in the control group (Tab. 2).

Type IV collagen immunoreactivity

Type IV collagen was immunolocalized to granulosa cells, theca cell compartment and stroma for all the follicle stages (Fig. 2). According to the results of statistical analysis, Type IV collagen immunoreactivity in primary follicles containing granulosa cells showed a significant increase in D1 and D3 groups as compared to the control group. The D2 group was similar to the control group. A significant increase was observed in the granulosa layer of secondary follicles in D1, D2 and D3 groups as compared to the control group. In theca layer, a significant increase was observed in D1 and D2 groups as compared to the control group. The D3 group was similar to the control group. In the tertiary follicles, there was a significant increase in the granulosa layer in D1, D2 and D3 groups as compared to the control group. D1 was the only group to show a significant increase in the theca layer as compared to the control group, whereas D2 and D3 groups were similar to the control group. According to these data, as the diabetes progressed and follicle developed in rats, type IV collagen immunoreactivity was observed to be increased in granulosa cells, whereas the theca layer immunoreactivity was observed to be similar to that in the control group (Tab. 3).

\section{Integrin $\alpha 3 \beta 1$ immunoreactivity}

Integrin $\alpha 3 \beta 1$ was immunolocalized to granulosa cells, theca cell compartment and stroma for all follicular stages (Fig. 3). According to the results of statistical analysis, $\alpha 3 \beta 1$ integrin immunoreactivity in primary and secondary follicles containing granulosa layer and secondary follicle theca layer showed a significant increase in D1 group, whereas no significant change was observed in D2 and D3 groups as compared to the control group. In D1 and D3 groups, the tertiary follicle granulosa layer showed a significant increase compared to the control group, whereas the D2 group was similar to the control group. There was a significant increase in tertiary follicle theca layer in D1, D2 and D3 groups as compared to the control group. According to these data, while the increase in $\alpha 3 \beta 1$ integrin immunoreactivity in granulosa and theca layers was significant in the first month of diabetes and in the initial stages of folliclar development in rats, this increase was observed not to be significant as diabetes progressed. However, there was a significant increase in $\alpha 3 \beta 1$ integrin immunoreactivity in the theca layer of follicles in the advanced stage of development as compared to the control group (Tab. 4).

\section{Discussion}

Diabetes has a negative effect on ovarian follicular development, and increases the morbidity of infertility (15-17). However, the mechanisms responsible for the pathogenesis of diabetesinduced infertility are not fully clear (17). Folliculogenesis and steroidogenesis deterioration, anovulation and stromal fibrosis may develop due to hyperglycemia $(18,19)$. It is very important to elucidate the molecular mechanisms underlying such diabetic disorders. Extracellular matrix (ECM) is a macromolecular network found in a three-dimensional form and without cells (20). ECM components are found in the ovary, follicular basement membrane, around follicular cells, and in the follicular fluid (6). The changes in the ECM expression in the normal state and in the state of diabetes inside the follicle, as well as the role of these changes in the regulation of follicular development are very important. Studies conducted on the expression and function of ECM proteins in ovarian tissue are quite limited in the case of diabetes. 
$340-347$

In this study, we aimed to investigate the immunohistochemical changes of ECM proteins such as laminin, type IV collagen and cell adhesion molecule $\alpha 3 \beta 1$ integrin in ovarian follicles at different stages of the development of diabetes in rats. In the light of available data, we would like to discuss whether the immunoreactivity of laminin, type IV collagen and $\alpha 3 \beta 1$ integrin, functions of these proteins, and their effects on follicular growth in ovarian granulosa and theca cells change with diabetes.

Although the molecules present in the ECM are classified in many ways, it includes molecules such as collagen, elastin, and proteoglycan, which are generally found to be structural, as well as some specialized structures such as integrin, fibronectin, etc. (20). Integrins are an important adhesion protein family (21). Integrin molecules and other receptors play a role in the transfer of ECM signals of cells to the intracellular skeletal structure. Ligand structures for integrins can be expressed as collagen, laminin and fibronectin. Interactions of these ligands and integrin help in the regulation of processes such as cell survival, cell migration and proliferation. Thus, the ECM components play a significant role in the ovarian function $(20,22,23)$.

Little is known regarding the stages of folliculogenesis and integrins in oogenesis (especially $\alpha 3 \beta 1$ integrin). Clearly, their function is thought to be more complex than a simple adhesion receptor or a signaling molecule that stimulates proliferation or differentiation in case of contact with ECM (24). In many cases, it is known that they primarily regulate events mediated by other integrins and even other non-integrin adhesion systems (24). Integrin $\alpha 3 \beta 1$ is expressed in granulosa cells throughout the follicular development and atresia in sheep. Ovine granulosa cells express integrin receptors for laminin $(\alpha 6 \beta 1, \alpha 3 \beta 1)$ and collagen $(\alpha 3 \beta 1)$, suggesting that these integrins can mediate in vivo activities of ECM components (7). In addition, in another study conducted on integrin subtype $\alpha 6 \beta 1$ integrin, it was reported that laminin- $\alpha 6 \beta 1$ integrin interaction increased the cell survival and proliferation and modulated the steroidogenesis of ovine granulosa cells (25).

Type VI collagen, which is one of the main structural components of ECM, plays a role in determining the organization and architecture of ECM (26), whereas, laminin glycoprotein allows the attachment of epithelial cells in the tissue and provides stability to the basal lamina structure with the associated type IV collagen $(20,27)$. ECM is also important for granulosa cell proliferation during folliculogenesis. In in vitro studies, the presence of ECM supports granulosa cell growth and proliferation (4). The effect of ECM proteins on granulosa cell proliferation depends on the size of follicle, and this suggests that the ECM composition in each follicle changes during the folliculogenesis (4). We observed that the expression of ECM proteins, which we investigated in the theca and granulosa layers of follicles in different developmental stages, changed in our study.

In the literature, laminin was immunoreactive in primary, secondary and antral follicles in granulosa and theca cells and was stained darker in antral follicles. Type IV collagen showed a low-level expression in granulosa cells, whereas it was abundant in the theca cell layer. The collagen distribution was consistent throughout the follicle maturation (6). In another study, type IV collagen expression was observed only in the theca layer of follicles and there was no expression observed in granulosa layer (16).

In our study, there was a significant increase in laminin, type VI collagen and $\alpha 3 \beta 1$ integrin expressions in all follicle types in both theca and granulosa layers compared to the control group in the first month of diabetes (in group D1). There was an increase observed in the immunoreactivity of laminin and type IV collagen in D2 and D3 groups. This increase was significant in some follicular stages and layers, but insignificant in others.

It was noteworthy that integrin expression did not change in the newly formed follicles (primary and secondary follicles) in the D2 and D3 groups, however, it tended to change and increase in the developing follicles (tertiary follicles). Also, there was a significant increase in $\alpha 3 \beta 1$ integrin immunoreactivity in all stages of diabetes in the theca layer of tertiary follicle.

Studies have reported differences in the number of ovarian follicles in the case of diabetes. Tatewaki et al (28) observed a reduction in the percentages of primary follicles in diabetic mice (28). In another study, the mean primordial, secondary and tertiary follicle counts decreased significantly in diabetic rats compared to the control group, whereas, atretic follicles increased significantly in diabetic rats (29).

Along with diabetes, the changes in the expression of laminin, type IV collagen and $\alpha 3 \beta 1$ integrin, which are ECM proteins inside the follicle, show that diabetes plays a role in the regulation of follicular development. Furthermore, it is thought that cellular interactions with ECM proteins through $\alpha 3 \beta 1$ integrin may be impaired with diabetes. In order to better understand the role of ECM in the effects of diabetes on the ovary, studies need to be conducted to elucidate the molecular mechanisms in folliculogenesis.

\section{References}

1. Maritim AC, Sanders RA, Watkins JB. Diabetes, Oxidative Stress, and Antioxidants: A Review. J Biochem Mol Toxic 2003; 17 (1): 24-38.

2. Shima H, Naeem E, Hassan M, Hossein N. Aloe Vera Gel Protects Ovarian Structure in Diabetic Rat. Am-Euras. J. Toxicol. Sci 2011; 3 (3): 197-203.

3. Burul-Bozkurt N, Pekiner C, Kelicen P. Diabetes alters aromatase enzyme levels in gonadal tissues of rats. Naunyn Schmiedebergs Arch Pharmacol 2010; 382: 33-41.

4. Woodruff TK, Shea LD. The role of the extracellular matrix in ovarian follicle development. Reprod Sci 2007; 14:6-10.

5. Rodgers RJ, van Wezel IL, Irving-Rodgers HF, Lavranos TC, Irvine CM, Krupa M. Roles of extracellular matrix in follicular development. J Reprod Fertil Suppl 1999; 54: 343-352.

6. Berkholtz CB, Lai BE, Woodruff TK, Shea LD. Distribution of extracellular matrix proteins type I collagen, type IV collagen, fibronectin, and laminin in mouse folliculogenesis. Histochem Cell Biol 2006; 126 : 583-592.

7. Monniaux D, Huet-Calderwood C, Le Bellego F, Fabre S, Monget P, Calderwood DA. Integrins in the Ovary. Semin Reprod Med 2006; 24 (4): 251-261.

8. Hynes RO. Integrins: Versatility, modulation, and signaling in cell adhesion. Cell 1992; 69 (1): 11-25. 
9. Miles AJ, Knutson JR, Skubitz APN, Furcht LT, McCarthy JB, Fields GB. A peptide model of basement membrane collagen a1 (IV) $531-543$ binds the $\alpha 3 \beta 1$ integrin. J Biol Chem 1995; 270: $29047-$ 29050.

10. Gehlsen KR, Dickerson K, Argraves WS, Engvall E, Rouslahti E. Subunit structure of a laminin-binding integrin and localization of its binding site on laminin. J Biol Chem 1989; 264: 19034-19038.

11. Wayner EA, Carter WG. Identification of multiple cell adhesion receptors for collagen and fibronectin in human fibrosarcoma cells possessing unique alpha and common b subunits. J Biol Chem 1987; 105: 1873-1884.

12. Regoli M, Bendayan M. Alterations in the expression of the $\alpha 3 \beta 1$ integrin in certain membrane domains of the glomerular epithelial cells (podocytes) in diabetes mellitus. Diabetologia 1997; 40: 15-22.

13. Yildirim AB, Ozdamar S, Yalcin B, Karabulut D. Changes in MAP2 and GFAP Immunoreactivity in Pup Hippocampus during Prepubertal and Pubertal Periods Caused by Maternal Subclinical Hypothyroidism. Eur J Anat 2019; 23 (1): 27-40.

14. Bernal AB, Vickers MH, Hampton MB, Poynton RA, Sloboda DM. Maternal undernutrition significantly impacts ovarian follicle number and increases ovarian oxidative stress in adult rat offspring. PLoS One 2010; 5 (12): e15558.

15. Tavakolian AA, Nouri M, Tavakolian AS. Surprisingly low infertility rate in married type 2 diabetic women: A rather curious paradox to the current opinion of insulin resistance as the joint pathogenesis of poly cystic ovary syndrome and type 2 diabetes mellitus. Diabetes Metab Syndr 2015; 9: 201-204.

16. Nandi A, Poretsky L. Diabetes and the female reproductive system. Endocrinol Metab Clin North Am 2013; 42: 915-946.

17. Wu Y, Li Y, Liao X, et al. Diabetes induces abnormal ovarian function via triggering apoptosis of granulosa cells and suppressing ovarian angiogenesis. Int J Biol Sci 2017; 13 (10): 1297-1308.
18. Gales C, Zamfir C, Radulescu D, Stoica B, Nechifor M. Protective effect of magnesium and metformin on endometrium and ovary in experimental diabetes mellitus. Magnes Res 2014; 27 (2): 69-76.

19. Yildirim N, Kose S, Yildirim AGS, Sahin C, Yigitturk G, Yavasoglu A, Erbas O. Silymarin ameliorates uterine and ovarian damage in streptozotocin induced diabetic rat model. Indian J Biochem Biophys 2018; 55: 137-142.

20. Cenan RS, Ergin E, Ekici Y. Structural and Functional Properties of the Extracellular Matrix. J Lit Pharm Sci 2018; 7 (3): 251-260.

21. Ruoslahti E. Integrins as signaling molecules and targets for tumor therapy. Kidney Int 1997; 51: 1413-1417.

22. Tanzer ML. Current concepts of extracellular matrix. J Orthop Sci 2006; 11 (3): 326-331.

23. Yurchenco PD. Basement membranes:cell scaffoldings and signaling platforms. Cold Spring Harb Perspect Biol 2011; 3 (2): a00491.

24. Kreidberg JA. Functions of $\alpha 3 \beta 1$ integrin. Curr Opin Cell Biol 2000; 12: 548-553.

25. Le Bellego F, Pisselet C, Huet C, Monget P, Monniaux D. Lamininalpha6 beta1 integrin interaction enhances survival and proliferation and modulates steroidogenesis of ovine granulosa cells. J Endocrinol 2002; 172: $45-59$.

26. Iwahashi M, Muragaki Y, Ooshima A, Nakano R. Type VI collagen expression during growth of human ovarian follicles. Fertil Steril 2000; 74: 343-347.

27. Theocharis AD, Skandalis SS, Gialeli C, Karamanos NK. Extracellular matrix structure. Adv Drug Deliv Rev 2016; 97: 4-27.

28. Tatewakil R, Otani H, Tanaka O, Kitadal J. A morphological study on the reproductive organs as a possible cause of developmental abnormalities in diabetic NOD mice. Histol Histopathol 1989; 4: 343-358.

29. Shima H, Naeem E, Hassan M, Hossein N. Aloe Vera Gel Protects Ovarian Structure in Diabetic Rat. Am-Euras. J. Toxicol. Sci 2011; 3 (3): 197-203. 